\title{
Prognostic Value of Blood Urea Nitrogen to Serum Albumin Ratio in Intensive Care Unit Patients with Lung Cancer
}

\section{Xiulan Peng ${ }^{1} *$ \\ Yali Huang ${ }^{1, *}$ \\ Haifeng $\mathrm{Fu}^{2, *}$ \\ Zhi Zhang ${ }^{3}$ \\ Anbing $\mathrm{He}^{\prime}$ \\ Renfeng Luo ${ }^{4}$}

'Department of Oncology, The Second Affiliated Hospital of Jianghan University, Wuhan, Hubei, People's Republic of China; ${ }^{2}$ Department of

Hepatopancreatobiliary Surgery, Sinopharm Dongfeng General Hospital, Hubei University of Medicine, Wuhan, Hubei, People's Republic of China; ${ }^{3}$ Department of Intensive Care Unit, The Second Affiliated Hospital of Jianghan University, Wuhan, Hubei, People's Republic of China; ${ }^{4}$ Department of Diagnostics, JiangHan University, Wuhan, Hubei, People's Republic of China

*These authors contributed equally to this work
Background: We aimed to evaluate the prognostic ability of blood urea nitrogen (BUN) to serum albumin ratio (BAR) to predict in-hospital mortality in patients with lung cancer in the intensive care unit (ICU).

Methods: Medical Information Mart for Intensive Care IV (MIMIC-IV v1.0) database was used to identify patients who were diagnosed with lung cancer. The primary outcome was inhospital mortality. Multivariate COX regression was used to investigate the association between BAR and in-hospital mortality and propensity score matching (PSM) and inverse probability of treatment weighting (IPTW) were also used to ensure the robustness of our findings. eICU-CRD database (validation cohort) was also applied to validate our findings. Results: The optimal cut-off value for BAR was $6.8 \mathrm{mg} / \mathrm{g}$. Among 1202 patients who were diagnosed with lung cancer, 287 high-BAR group $(\geq 6.8 \mathrm{mg} / \mathrm{g})$ patients and 287 low-BAR group $(<6.8 \mathrm{mg} / \mathrm{g})$ patients, who had similar propensity scores were included in this study. After matching, the high-BAR group had significantly higher in-hospital mortality (hazard ratio, HR, 2.24, 95\% confidence index, 95\% CI, 1.57-3.19, $\mathrm{P}<0.001)$ even after adjustment for confounding factors. Moreover, the performance of BAR was superior to that of BUN and serum albumin alone and could add net benefit in predicting in-hospital mortality. Those results were further confirmed in the validation cohort.

Conclusion: As an easily accessible and cost-effective parameter, BAR could serve as a good prognostic predictor for lung cancer patients in ICU.

Keywords: blood urea nitrogen to serum albumin ratio, lung cancer, intensive care unit, Medical Information Mart for Intensive Care, eICU-CRD, prognosis

\section{Introduction}

Despite great progress in our understanding of risk, development, and treatment options, lung cancer, which usually consists of small cell lung cancer and non-small cell lung cancer, remains one of the most common diagnosed cancers and the leading cause of cancer-related death worldwide. ${ }^{1-4}$ Due to the nature of the disease and the aggressive treatments, lung cancer patients usually require admission to intensive care units (ICU) for invasive monitoring or treatment. ${ }^{5-7}$ Compared with other solid tumors, lung cancer patients admitted to ICU tend to have one of the poorest ICU and in-hospital survival rates. ${ }^{8-10}$ Hence, it is essential for clinicians to identify lung cancer patients at high risk of mortality.

Serum albumin is one of the most familiar nutritional indexes and has been demonstrated to be a prognostic factor for different types of cancers. ${ }^{11-14}$ Blood
Correspondence: Xiulan Peng Department of Oncology, The Fifth Hospital of Wuhan, The Second Affiliated Hospital of Jianghan University, 122 Xianzheng Road, Wuhan, Hubei, 430050, People's Republic of China Tel +86027-84830I20

Email pengxlwh@163.com 
urea nitrogen (BUN) is an important parameter reflecting the relationship between kidney condition and nutritional status of patients and has also been found to be associated with mortality. ${ }^{15}$ Moreover, the combination of serum albumin and bun, bun to serum albumin ratio (BAR), which is calculated from the quotient between BUN and albumin, was introduced as an important predictor of mortality in various diseases, including gastrointestinal bleeding, community-acquired pneumonia and so on. ${ }^{16-18}$ However, to the best of our knowledge, no study has been constructed to investigate the association between BAR and in-hospital mortality for critical care patients with lung cancer. Hence, in the current study, we initially investigated the correlation between BAR and prognosis of ICU patients with lung cancer using data from the Medical Information Mart for Intensive Care IV (MIMICIV version 1.0) database. Then, propensity score matching (PSM) and propensity score-based inverse probability of treatment weighting (IPTW) was introduced to ensure the robustness of our results, we further verified this finding in another big public database (eICU Collaborative Research Database, eICU-CRD v2.0).

\section{Materials and Methods}

\section{Study Population}

We obtained data from the MIMIC IV database and eICU-CRD database in accordance with the ethical standards of the Institutional Review Board (IRB) of the Massachusetts Institute of Technology (MIT). eICUCRD contains data of more than 200 thousand ICU admissions in 2014 and 2015 at 208 US hospitals while MIMIC-IV includes information of more than 70,000 patients admitted to the ICUs of Beth Israel Deaconess Medical Center in Boston, MA, from 2008 to $2019 .{ }^{19,20}$ This study was conducted in accordance with the Helsinki Declaration and authors had successfully accomplished the National Institutes of Health's (NIH) online training course and the Protection of Human Research Participants Examination and got permission to extract data from MIMIC IV and eICU databases. Moreover, the study protocol was reviewed and successfully approved by the Ethics Committee of the Second Affiliated Hospital of Jianghan University. Considering that this was a retrospective study and all patients in this study were extracted from public database, informed consent was waived.

\section{Selection of Participants}

Adult patients who were diagnosed with lung cancer based on the ninth or tenth revision of International Classification of Diseases (ICD-9/10) code during their admissions were included in this study. Moreover, for patients readmitted to the ICU, only the first ICU and first hospital admissions were included in this study. We also excluded patients with missing bun or serum albumin or who spent less than 48 hours in the ICU (Figure 1).

\section{Variable Extraction}

Baseline characteristics and admission information: age, gender, weight, tumor type, and severity score measured by the sequential organ failure assessment (SOFA) score, the Oxford acute severity of illness score (OASIS), acute physiology score III (APSIII) and the Charlson comorbidity score were calculated as described in previous studies. ${ }^{21-24}$ Comorbidities including hypertension, diabetes, chronic kidney disease (CKD), congestive heart failure (CHF), myocardial infarct and liver disease were also collected for analysis based on the (ICD-9/10) codes. Complications including sepsis based on sepsis 3.0 criteria, ${ }^{25}$ acute kidney injury based on KDIGO guideline in 48 hours, ${ }^{26}$ acute heart failure (AHF) and acute respiratory failure (ARF) based on ICD codes were also included in this study. Use of mechanical ventilation (MV), vasopressors and renal replacement therapy during their hospital stay were also recorded in this study. Moreover, initial vital signs and laboratory results were also extracted by structured query language with PostgreSQL 9.6.

The BAR ( $\mathrm{mg} / \mathrm{g}$ ) was calculated by initial serum BUN $(\mathrm{mg} / \mathrm{dL}) /$ serum albumin $(\mathrm{g} / \mathrm{dL})$.

The primary outcome in this study was in-hospital mortality.

\section{Statistical Analyses}

Continuous variables were expressed as mean (standardized mean difference, SMD), categorical covariates were reported as number (percentage). X-tile software (version 3.6.1) was applied to determine the best cutoff values for BAR in MIMIC-IV database. Then, clinical features between high BAR and low BAR groups were analyzed with either Student's $t$-test or Chi-squared test as appropriate. Propensity score matching (PSM) and propensity score-based inverse probability of treatment weighting (IPTW) were also applied to adjust the imbalance of the covariates between two groups to 


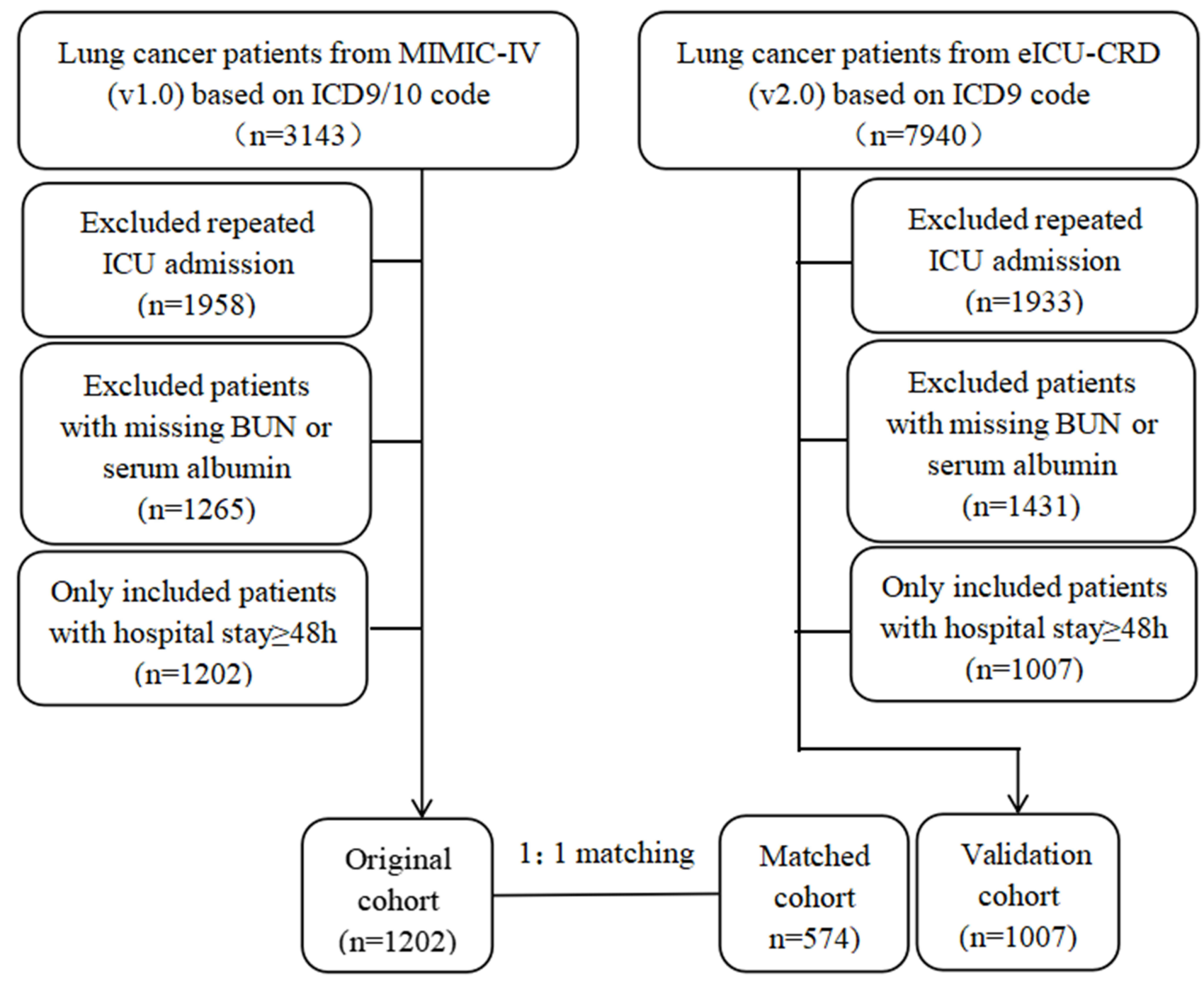

Figure I Study flow diagram of the present study.

ensure the robustness of our results. One-to-one nearest neighbor matching with a caliper width of 0.2 was applied in the current study. Multivariate COX regression and adjusted hazard ratio (HR) were also conducted in the original cohort, matched cohort, weighted cohort and validation cohort to investigate the association between BAR and in-hospital mortality. Receiver operating characteristic curve (ROC) was used to compare the predictive performance of BAR, BUN and albumin in predicting in-hospital mortality in different cohorts. Finally, the decision curve analysis (DCA) was also performed to evaluate the potential clinical usefulness and benefits of the GLR. All analyses were performed using $\mathrm{R}$ (version 4.1.0) and $\mathrm{X}$-tile (version 3.6.1) software and $\mathrm{p}<0.05$ was considered statistically significant.

\section{Results}

\section{Patient Clinical Features}

A total of 2209 patients (1202 patients in the original cohort, 1007 patients in the validation cohort) with lung cancer were analyzed in this study. The best cut-off value for BAR, determined using X-tile software, was $6.8 \mathrm{mg} / \mathrm{g}$. We grouped the patients according to the measurements of BAR. In the original cohort, compared to patients with low BAR $(<6.8 \mathrm{mg} / \mathrm{g})$, those with high BAR were older, higher proportion of males, had metastatic cancer and interventions during hospital stay, had more comorbidities and complications, had higher levels of severity scores, total bilirubin, white blood counts, anion gap, creatinine and potassium, and lower level of mean arterial pressure (MAP), hemoglobin, platelets and bicarbonate. Moreover, 
patients in high BAR group had prolonged ICU stays and higher in-hospital mortality. After PSM and IPTW, almost all covariates in the matched cohort and in the weighted cohort were balanced between two groups (Supplemental Figure 1). Moreover, patients in the validation cohort exhibited similar characteristics to those in the original cohort (Table 1).

\section{Prognostic Value of BAR for Primary Endpoint}

As described in Table 1, compared with patients in lowBAR group, high-BAR group had a relatively higher inhospital mortality in original cohort (38.7\% versus $14.2 \%$, $\mathrm{P}<0.001)$, in matched cohort $(29.6 \%$ versus $18.5 \%$, $\mathrm{P}=0.002)$ as well as in validation cohort $(38.2 \%$ versus $14.3 \%, \mathrm{P}<0.001)$. Those findings were further confirmed by Kaplan-Meier curves. As described in Figure2A-C, high BAR group had a poor in-hospital mortality in original cohort, in matched cohort and in validation cohort.

The univariate COX regression analysis indicated that high BAR group patients were associated with increased in-hospital mortality, with a crude hazard ratio (HR) of 2.60 (95\% confidence index, 95\% CI, 2.03-3.33, P<0.001) and the association remained robust after PSM (HR=2.04, 95\% CI 1.44-2.89, $\mathrm{P}<0.001)$ and IPTW $(\mathrm{HR}=2.00,95 \%$ CI $1.42-2.82, \mathrm{P}<0.001$ ) (Table 2). These findings were further confirmed by the results of the multivariate analyses. High BAR was still an independent predictor for inhospital mortality in original cohort $(\mathrm{HR}=2.09,95 \% \mathrm{CI}$ $1.58-2.76, \mathrm{P}<0.001)$, in matched cohort $(\mathrm{OR}=2.24,95 \%$ CI 1.57-3.19, $\mathrm{P}<0.001)$ and in weighted cohort $(\mathrm{OR}=2.77$, 95\% CI 2.03-4.58, $\mathrm{P}<0.001)$ after adjustment for age, gender, weight, tumor type, comorbidities, complications, score system, interventions, vital signs and laboratory results (Table 2). Moreover, patients in the validation cohort showed similar results, which indicated that BAR was a significant and robust predictor for in-hospital mortality in lung cancer patients in ICU.

To further investigate whether BAR remained a prognostic factor in certain patient subgroups, we performed exploratory subgroup analyses. Forest plot demonstrated that BAR was an independent prognostic factor in the original cohort in all subgroups (Figure 3A). Moreover, patients from the validation cohort showed similar results. BAR can predict patient survival in almost all subgroups except for patients with acute heart failure and chronic kidney disease (Figure 3B).

\section{Clinical Usefulness of BAR}

BAR exhibited better predictive abilities for in-hospital mortality when compared with BUN or serum albumin alone in the original cohort, in the matched cohort, as well as in the validation cohort (Figure 2D and Table 3).

A DCA curve was also introduced to evaluate the clinical use of BAR for in-hospital mortality. According to the DCA, when the threshold probability for a patient was within the range of $0-100 \%$, the BAR added more net benefit than the "treat all" or "treat none" strategies both in the original cohort, in the matched cohort, and in the validation cohort, which indicated that BAR could have clinical usefulness (Figure 4A-C).

\section{Discussion}

In the current study, we retrospectively enrolled 2209 ICU patients with lung cancer and found that high BAR group patients had increased in-hospital mortality and concluded that initial BAR could be an independent predictor for inhospital mortality after adjusting confounding factors. In addition, the predictive performance of BAR was superior to that of serum albumin or BUN alone and could add more net benefit in terms of in-hospital mortality rather than "treat all" or "treat none". Hence, those results suggested that BAR might be a good predictor for identifying patients at high risk of in-hospital mortality among lung cancer patients in ICU.

As one of the leading causes of cancer-related deaths all over the world, lung cancer patients often need invasive monitoring or treatment and have a relatively low survival rate, especially patients in ICU. ${ }^{9,27}$ Using the data from surveillance, epidemiology, and end results-medicare registry, Christopher et al demonstrated that the in-hospital mortality was $24 \%$ in patients with lung cancer who were admitted to an ICU for reasons other than surgical resection of their tumor. ${ }^{28}$ In the current study, the in-hospital mortality rate for ICU patients with lung cancer was $26.0 \%$ in the original cohort and $26.4 \%$ in the validation cohort.

The association between BAR and prognosis in patients with disease of the respiratory system has been investigated in previous studies. Seung et al conducted a retrospective study of 443 patients who were admitted to emergency department and concluded that BAR was a useful prognostic factor of 28-day mortality in aspiration pneumonia patients. ${ }^{29}$ A similar result has also been found in critically ill patients with acute pulmonary embolism. Using the data extracted from MIMIC-III, Fang et al 


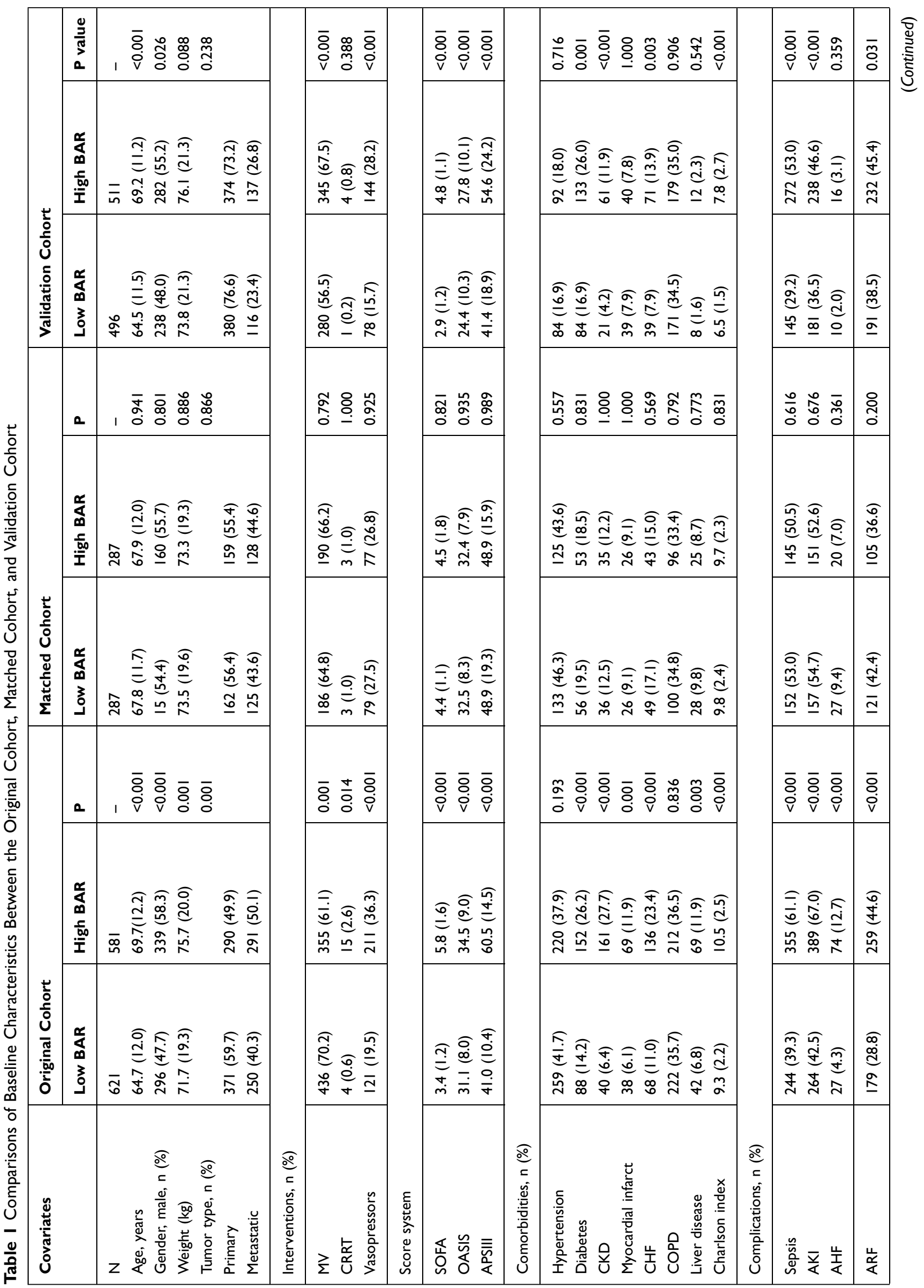








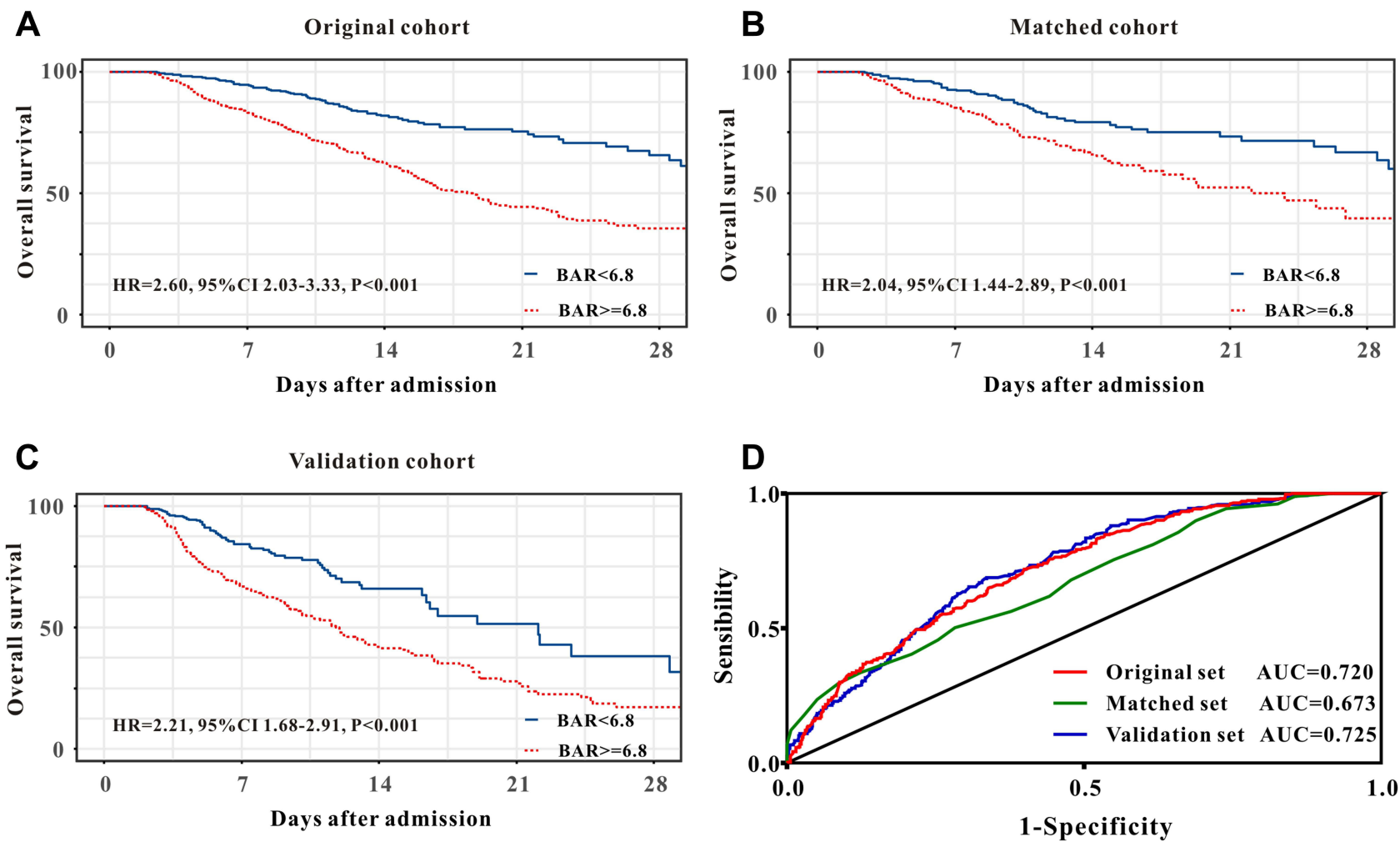

Figure 2 Kaplan-Meier curves for in-hospital survival for lung cancer patients stratified by BAR in the original cohort (A), in the matched cohort (B), and in the validation cohort (C). Receiver operating characteristic curve analysis of BAR for in-hospital mortality in the original cohort, in the matched cohort, and in the validation cohort (D).

investigated the correlation between BAR and prognosis of $1048 \mathrm{ICU}$ patients with acute pulmonary embolism and concluded that BAR was an independent predictor for ICU mortality as well as 28-day mortality after ICU admission and the predictive performance of BAR was superior to the SOFA score and APSIII score. ${ }^{30}$ Moreover, Huang et al conducted a retrospective, observational study of 602 patients and demonstrated that elevated BAR at admission is an independent risk factor for in-hospital mortality in coronavirus disease patients (2019). ${ }^{31}$ Our study added the evidence that BAR was a reliable predictor for prognosis of patients with disease of the respiratory system. We firstly found that initial BAR could be an independent predictor for in-hospital mortality even after adjusting confounding factors in patients with lung cancer who were admitted to ICU. Moreover, the predictive

Table 2 Summary of Results of Primary Outcome

\begin{tabular}{|c|c|c|c|c|c|c|c|c|}
\hline & \multicolumn{2}{|l|}{ Original Cohort } & \multicolumn{2}{|c|}{ Matched Cohort } & \multicolumn{2}{|c|}{ Weighted Cohort } & \multicolumn{2}{|c|}{ Validation Cohort } \\
\hline & HR (95\% CI) & $P$ value & HR (95\% CI) & $P$ value & HR (95\% CI) & $P$ value & HR (95\% Cl) & $P$ value \\
\hline Unadjusted & $2.60(2.03-3.33)$ & $<0.001$ & $2.04(1.44-2.89)$ & $<0.001$ & $2.00(1.42-2.82)$ & $<0.001$ & $2.21(1.68-2.91)$ & $<0.001$ \\
\hline Model I & $2.66(2.07-3.4 I)$ & $<0.001$ & $2.10(1.48-2.99)$ & $<0.001$ & $2.05(1.44-2.91)$ & $<0.001$ & $2.4 \mathrm{I}(1.83-3.17)$ & $<0.001$ \\
\hline Model 2 & $2.45(1.89-3.19)$ & $<0.001$ & $2.13(1.50-3.03)$ & $<0.001$ & $2.22(1.54-3.22)$ & $<0.001$ & $2.36(1.79-3.10)$ & $<0.001$ \\
\hline Model 3 & $2.04(1.56-2.69)$ & $<0.001$ & $2.28(1.60-3.25)$ & $<0.001$ & $2.40(1.63-3.52)$ & $<0.001$ & $2.30(1.75-3.02)$ & $<0.001$ \\
\hline Model 4 & $2.09(1.58-2.76)$ & $<0.001$ & $2.24(1.57-3.19)$ & $<0.001$ & $2.77(2.03-4.58)$ & $<0.001$ & $2.20(1.67-2.90)$ & $<0.001$ \\
\hline
\end{tabular}

Notes: Model I adjusted for age, gender, weight, tumor type. Model 2 adjusted for model I plus comorbidities and complications. Model 3 adjusted for model 2 plus score system, interventions and Charlson index. Model 4 adjusted for model 3 plus vital signs and laboratory results.

Abbreviations: $\mathrm{HR}$, hazard ratio; $95 \% \mathrm{Cl}, 95 \%$ confidence index. 

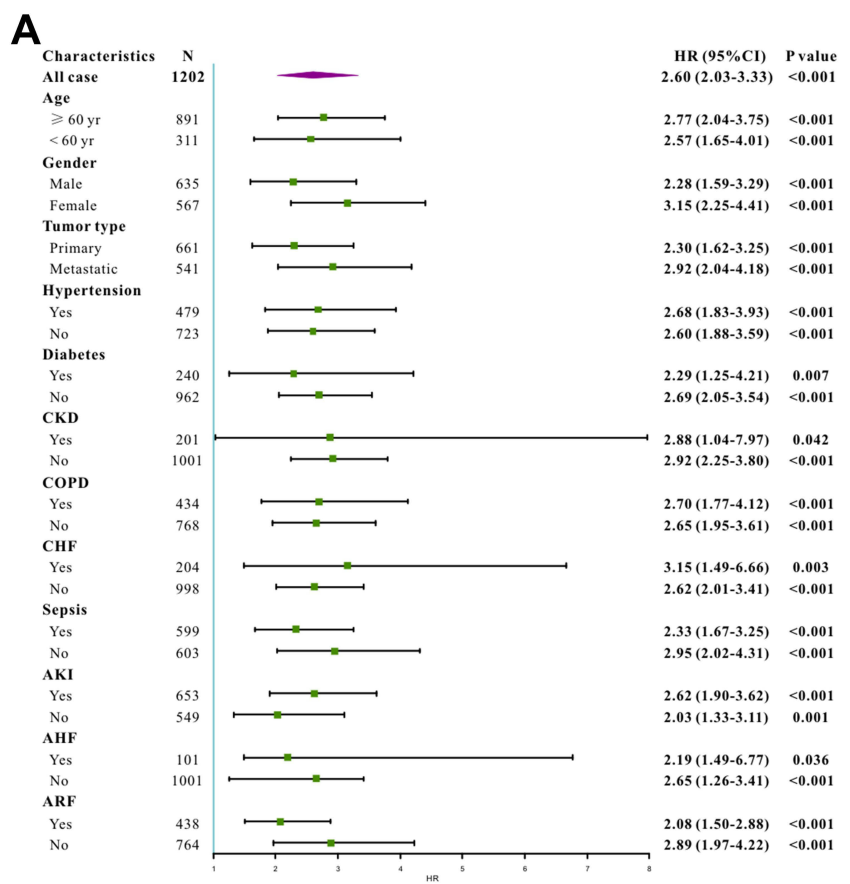

B

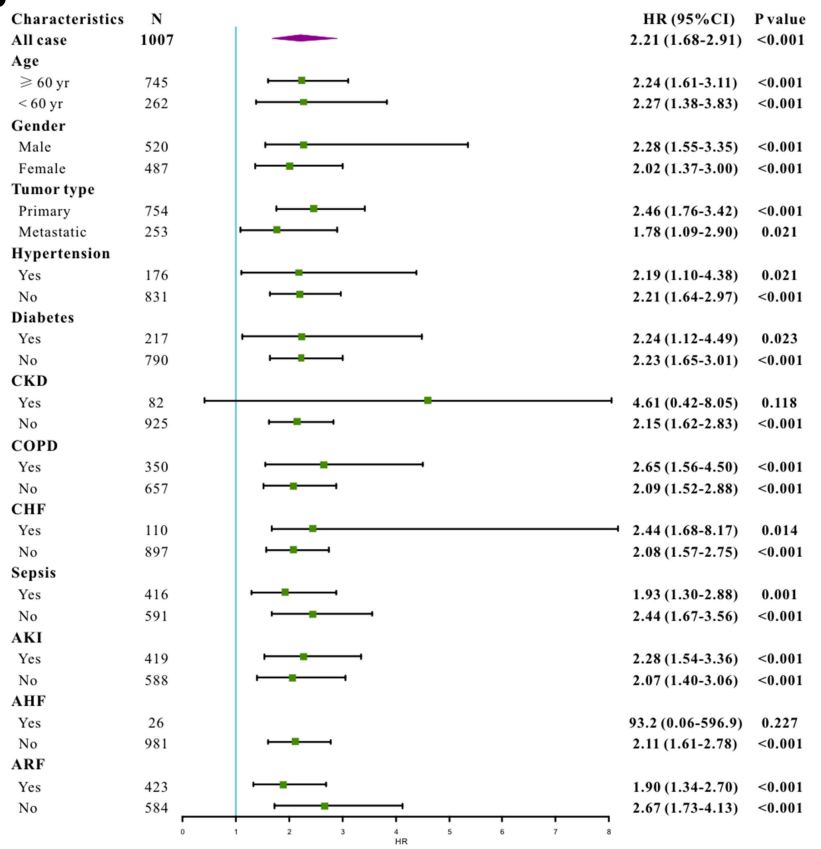

Figure 3 Subgroup analysis for hazard ratio of lung cancer patients with BAR $\geq 6.8 \mathrm{mg} / \mathrm{g}$ versus BAR $<6.8 \mathrm{mg} / \mathrm{g}$ in different groups in the original cohort (A) and in the validation cohort $\mathbf{( B )}$.

performance of BAR was superior to that of serum albumin or BUN alone and could add more net benefit in terms of in-hospital mortality rather than "treat all" or "treat none".

A high BAR represents a high BUN concentration and a low albumin level. As a well-known index of renal function, BUN could also reflect the complex mutuality among nutritional status, protein metabolism, and renal condition of the patient. $^{32,33}$ Increasing studies have focused attention on the BUN-based index or BUN alone for the prognosis of patients with different types of diseases, such as hemodialysis, ${ }^{34} \mathrm{CKD},{ }^{35}$ peripheral arterial disease, ${ }^{36}$ and so on. Serum albumin plays an important role in immune-modulation, anti-oxidation, antiinflammatory and endothelial stabilization. ${ }^{37,38}$ Moreover, serum albumin levels could be decreased by the status of malnutrition, inflammation, hepatocellular injury, renal losses, and so on. ${ }^{39}$ Previous studies also demonstrated

Table 3 Receiver Operating Curve Analysis

\begin{tabular}{|c|c|c|c|c|}
\hline Variable & Sensitivity & Specificity & AUC (95\% Cl) & $P$ value \\
\hline \multicolumn{5}{|c|}{ Original cohort } \\
\hline BAR & 71.9 & 60.1 & $0.720(0.694-0.745)$ & \\
\hline BUN & 62.9 & 62.0 & $0.660(0.633-0.687)$ & $<0.001$ \\
\hline Albumin & 51.8 & 73.6 & $0.672(0.646-0.697)$ & $<0.001$ \\
\hline \multicolumn{5}{|c|}{ Matched cohort } \\
\hline BAR & 71.7 & 50.2 & $0.673(0.633-0.7 \mathrm{II})$ & \\
\hline BUN & 94.2 & 17.2 & $0.532(0.490-0.574)$ & 0.003 \\
\hline Albumin & 85.5 & 35.1 & $0.613(0.57 \mid-0.653)$ & 0.046 \\
\hline \multicolumn{5}{|c|}{ Validation cohort } \\
\hline BAR & 68.8 & 66.4 & $0.725(0.697-0.753)$ & \\
\hline BUN & 74.1 & 50.1 & $0.649(0.619-0.679)$ & $<0.001$ \\
\hline Albumin & 58.3 & 79.6 & $0.686(0.659-0.711)$ & 0.001 \\
\hline
\end{tabular}

Abbreviations: BAR, blood urea nitrogen to serum albumin ratio; BUN, blood urea nitrogen; AUC, area under the receiver operating curve. 

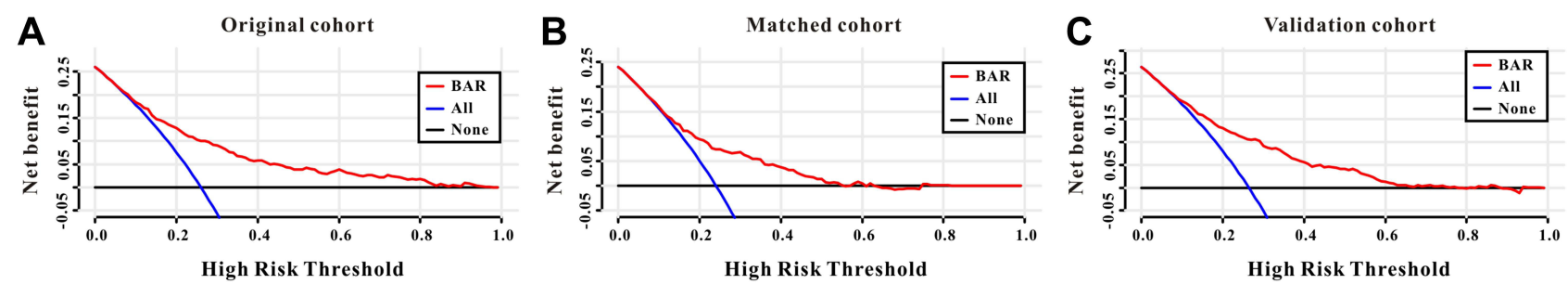

Figure 4 Decision curve analysis of BAR for in-hospital mortality in lung cancer patients to detect its clinical usefulness in the original cohort (A), in the matched cohort (B), and in the validation cohort (C).

some albumin-based ratio for the diagnostic and prognostic of lung cancer. ${ }^{40-42}$ However, to the best of our knowledge, limited data are available on the combination of BUN and serum albumin for the prognosis of patients with lung cancer considering that the increased BUN and decreased serum albumin levels are in agreement with previous studies. In the current study, we retrospectively enrolled 2209 ICU patients with lung cancer using two large free public databases and noted a positive correlation between BAR and the prognosis of patients. This association became robust even after PSM and IPTW were also employed to eliminate the imbalance of the covariates between high-BAR and low-BAR groups. Furthermore, this association between BAR and in-hospital mortality was further confirmed in another big database with 1007 ICU patients with lung cancer.

Despite the relatively large sample size, there were still some limitations observed in this study. Firstly, we only calculated the initial BAR after their ICU admission but did not assess changes in BAR in any patients during hospital stay. Values of serum albumin or BUN may vary over time, and dynamic monitoring of these values may be more accurate. Secondly, we did not obtain levels of serum C-reactive protein and other inflammatory or nutritional indicators, which may be helpful to investigate the mechanism of the association between BAR and prognosis of lung cancer patients. Finally, this was a retrospective study, further prospective multicenter studies are proposed to validate the conclusions of our study and to investigate the potential mechanism behind them.

\section{Conclusion}

In the current study, we firstly demonstrated that initial BAR could serve as an independent prognostic predictor of in-hospital mortality in lung cancer patients in ICU, with good discrimination and clinical usefulness. BAR, which is an easily accessible and cost-effective parameter, provides a helpful index for clinicians to stratify the risk of mortality. Despite the solid statistics of this study, the findings of this study need more validations.

\section{Data Sharing Statement}

All data in our study are available from the corresponding author upon reasonable request.

\section{Statement of Ethics}

This study was conducted in accordance with the Helsinki Declaration and authors had successfully accomplished the National Institutes of Health's (NIH) online training course and the Protection of Human Research Participants Examination and got the permission to extract data from MIMIC IV and eICU databases. Moreover, the study protocol was reviewed and successfully approved by the Ethics Committee of the Second Affiliated Hospital of Jianghan University and informed consent was waived.

\section{Funding}

This study was supported by Wuhan Municipal Health Commission (No: WX21D42).

\section{Disclosure}

The authors declare that they have no competing interests.

\section{References}

1. Bade BC, Dela CC. Lung cancer 2020: epidemiology, etiology, and prevention. Clin Chest Med. 2020;41:1-24. doi:10.1016/j. ccm.2019.10.001

2. Mao Y, Yang D, He J, Krasna MJ. Epidemiology of lung cancer. Surg Oncol Clin N Am. 2016;25:439-445. doi:10.1016/j.soc.2016.02.001

3. Schabath MB, Cote ML. Cancer progress and priorities: lung cancer. Cancer Epidemiol Biomarkers Prev. 2019;28:1563-1579. doi:10.1158/ 1055-9965.EPI-19-0221

4. Nasim F, Sabath BF, Eapen GA. Lung cancer. Med Clin North Am. 2019;103:463-473. doi:10.1016/j.mena.2018.12.006

5. Garcia-Suarez J, de la Cruz J, Cedillo A, et al. Impact of hematologic malignancy and type of cancer therapy on COVID-19 severity and mortality: lessons from a large population-based registry study. J Hematol Oncol. 2020;13:133. doi:10.1186/s13045-020-00970-7 
6. Martos-Benitez FD, Soto-Garcia A, Gutierrez-Noyola A. Clinical characteristics and outcomes of cancer patients requiring intensive care unit admission: a prospective study. J Cancer Res Clin Oncol. 2018;144:717-723. doi:10.1007/s00432-018-2581-0

7. Ostermann M, Ferrando-Vivas P, Gore C, Power S, Harrison D. Characteristics and outcome of cancer patients admitted to the ICU in England, Wales, and Northern Ireland and national trends between 1997 and 2013. Crit Care Med. 2017;45:1668-1676. doi:10.1097/ CCM.0000000000002589

8. Puxty K, McLoone P, Quasim T, Kinsella J, Morrison D. Survival in solid cancer patients following intensive care unit admission. Intensive Care Med. 2014;40:1409-1428. doi:10.1007/s00134-014-3471-9

9. Andrejak C, Terzi N, Thielen S, et al. Admission of advanced lung cancer patients to intensive care unit: a retrospective study of 76 patients. BMC Cancer. 2011;11:159. doi:10.1186/1471-2407-11-159

10. Puxty K, Grant $\mathrm{CH}$, McLoone P, et al. Factors associated with intensive care admission in patients with lung cancer: a population-based observational study of 26,731 patients. $B M C$ Pulm Med. 2020;20:36. doi:10.1186/s12890-020-1071-8

11. Yang N, Han X, Yu J, Shu W, Qiu F, Han J. Hemoglobin, albumin, lymphocyte, and platelet score and neutrophil-to-lymphocyte ratio are novel significant prognostic factors for patients with small-cell lung cancer undergoing chemotherapy. $J$ Cancer Res Ther. 2020;16:1134-1139. doi:10.4103/jcrt.JCRT_1066_19

12. Ouyang X, Dang Y, Zhang F, Huang Q. Low serum albumin correlates with poor survival in gastric cancer patients. Clin Lab. 2018;64:239-245. doi:10.7754/Clin.Lab.2017.170804

13. Sun H, He B, Nie Z, et al. A nomogram based on serum bilirubin and albumin levels predicts survival in gastric cancer patients. Oncotarget. 2017;8:41305-41318. doi:10.18632/oncotarget.17181

14. Kao HK, Lofstrand J, Loh CY, et al. Nomogram based on albumin and neutrophil-to-lymphocyte ratio for predicting the prognosis of patients with oral cavity squamous cell carcinoma. Sci Rep. 2018;8:13081. doi:10.1038/s41598-018-31498-z

15. Liu J, Sun LL, Wang J, Ji G. Blood urea nitrogen in the prediction of in-hospital mortality of patients with acute aortic dissection. Cardiol J. 2018;25:371-376. doi:10.5603/CJ.a2017.0075

16. Bae SJ, Kim K, Yun SJ, Lee SH. Predictive performance of blood urea nitrogen to serum albumin ratio in elderly patients with gastrointestinal bleeding. Am J Emerg Med. 2021;41:152-157. doi:10.1016/ j.ajem.2020.12.022

17. Bae SJ, Lee SH, Yun SJ, Kim K. Comparison of IVC diameter ratio, $\mathrm{BUN} /$ creatinine ratio and BUN/albumin ratio for risk prediction in emergency department patients. Am J Emerg Med. 2021;47:198-204. doi:10.1016/j.ajem.2021.03.081

18. Ugajin M, Yamaki K, Iwamura N, Yagi T, Asano T. Blood urea nitrogen to serum albumin ratio independently predicts mortality and severity of community-acquired pneumonia. Int J Gen Med. 2012;5:583-589. doi:10.2147/IJGM.S33628

19. Pollard TJ, Johnson A, Raffa JD, Celi LA, Mark RG, Badawi O. The eICU collaborative research database, a freely available multi-center database for critical care research. Sci Data. 2018;5:180178. doi:10.1038/sdata.2018.178

20. Johnson ABLP. MIMIC-IV (Version 1.0). PhysioNet; 2021.

21. Raith EP, Udy AA, Bailey M, et al. Prognostic accuracy of the SOFA score, SIRS criteria, and qSOFA score for in-hospital mortality among adults with suspected infection admitted to the intensive care unit. JAMA. 2017;317:290-300. doi:10.1001/jama.2016.20328

22. Johnson AE, Kramer AA, Clifford GD. A new severity of illness scale using a subset of Acute Physiology and Chronic Health Evaluation data elements shows comparable predictive accuracy. Crit Care Med. 2013;41:1711-1718. doi:10.1097/CCM.0b013e31828a24fe

23. Knaus WA, Wagner DP, Draper EA, et al. The APACHE III prognostic system. Risk prediction of hospital mortality for critically ill hospitalized adults. Chest. 1991;100:1619-1636. doi:10.1378/ chest.100.6.1619
24. Quan H, Sundararajan V, Halfon P, et al. Coding algorithms for defining comorbidities in ICD-9-CM and ICD-10 administrative data. Med Care. 2005;43:1130-1139. doi:10.1097/01. mlr.0000182534.19832.83

25. Singer M, Deutschman CS, Seymour CW, et al. The third international consensus definitions for sepsis and septic shock (Sepsis-3). JAMA. 2016;315:801-810. doi:10.1001/ jama.2016.0287

26. Kellum JA, Lameire N. Diagnosis, evaluation, and management of acute kidney injury: a KDIGO summary (Part 1). Crit Care. 2013;17:204. doi:10.1186/cc11454

27. Bonomi MR, Smith CB, Mhango G, Wisnivesky JP. Outcomes of elderly patients with stage IIIB-IV non-small cell lung cancer admitted to the intensive care unit. Lung Cancer. 2012;77:600-604. doi:10.1016/j.lungcan.2012.05.103

28. Slatore CG, Cecere LM, Letourneau JL, et al. Intensive care unit outcomes among patients with lung cancer in the surveillance, epidemiology, and end results-medicare registry. $J$ Clin Oncol. 2012;30:1686-1691. doi:10.1200/JCO.2011.40.0846

29. Ryu S, Oh SK, Cho SU, et al. Utility of the blood urea nitrogen to serum albumin ratio as a prognostic factor of mortality in aspiration pneumonia patients. Am $J$ Emerg Med. 2021;43:175-179. doi:10.1016/j.ajem.2020.02.045

30. Fang J, Xu B. Blood urea nitrogen to serum albumin ratio independently predicts mortality in critically ill patients with acute pulmonary embolism. Clin Appl Thromb Hemost. 2021;27:1319775551. doi:10.1177/10760296211010241

31. Kucukceran K, Ayranci MK, Girisgin AS, Kocak S, Dundar ZD. The role of the BUN/albumin ratio in predicting mortality in COVID-19 patients in the emergency department. Am $J$ Emerg Med. 2021;48:33-37. doi:10.1016/j.ajem.2021.03.090

32. Huang D, Yang $\mathrm{H}, \mathrm{Yu} \mathrm{H}$, et al. Blood urea nitrogen to serum albumin ratio (BAR) predicts critical illness in patients with Coronavirus disease 2019 (COVID-19). Int J Gen Med. 2021;14:4711-4721. doi:10.2147/IJGM.S326204

33. Ok F, Erdogan O, Durmus E, Carkci S, Canik A. Predictive values of blood urea nitrogen/creatinine ratio and other routine blood parameters on disease severity and survival of COVID-19 patients. J Med Virol. 2021;93:786-793. doi:10.1002/jmv.26300

34. Tanaka S, Ninomiya $\mathrm{T}$, Taniguchi $\mathrm{M}$, et al. Impact of blood urea nitrogen to creatinine ratio on mortality and morbidity in hemodialysis patients: the Q-Cohort study. Sci Rep. 2017;7:14901. doi:10.1038/s41598-017-14205-2

35. Seki M, Nakayama M, Sakoh T, et al. Blood urea nitrogen is independently associated with renal outcomes in Japanese patients with stage 3-5 chronic kidney disease: a prospective observational study. BMC Nephrol. 2019;20:115. doi:10.1186/ s12882-019-1306-1

36. Gary T, Pichler M, Schilcher G, et al. Elevated blood urea nitrogen is associated with critical limb ischemia in peripheral arterial disease patients. Medicine (Baltimore). 2015;94:e948. doi:10.1097/ MD.0000000000000948

37. Caraceni P, Domenicali M, Tovoli A, et al. Clinical indications for the albumin use: still a controversial issue. Eur J Intern Med. 2013;24:721-728. doi:10.1016/j.ejim.2013.05.015

38. Nicholson JP, Wolmarans MR, Park GR. The role of albumin in critical illness. Br J Anaesth. 2000;85:599-610. doi:10.1093/bja/ 85.4.599

39. Hedlund JU, Hansson LO, Ortqvist AB. Hypoalbuminemia in hospitalized patients with community-acquired pneumonia. Arch Intern Med. 1995;155:1438-1442. doi:10.1001/archinte.1995. 00430130132014

40. Ni XF, Wu J, Ji M, et al. Effect of C-reactive protein/albumin ratio on prognosis in advanced non-small-cell lung cancer. Asia Pac J Clin Oncol. 2018;14:402-409. doi:10.1111/ajco.13055 
41. Guo X, Shao J, Zhai B, et al. Relationship and prognostic significance between preoperative serum albumin to globulin ratio and CT features of non-small cell lung cancer. Eur $J$ Radiol. 2020;128:109039. doi:10.1016/j.ejrad.2020.109039
42. Yang JR, Xu JY, Chen GC, et al. Post-diagnostic C-reactive protein and albumin predict survival in Chinese patients with non-small cell lung cancer: a prospective cohort study. Sci Rep. 2019;9:8143. doi:10.1038/s41598-019-44653-x

\section{Publish your work in this journal}

The International Journal of General Medicine is an international, peer-reviewed open-access journal that focuses on general and internal medicine, pathogenesis, epidemiology, diagnosis, monitoring and treatment protocols. The journal is characterized by the rapid reporting of reviews, original research and clinical studies across all disease areas. The manuscript management system is completely online and includes a very quick and fair peer-review system, which is all easy to use. Visit http://www.dovepress.com/ testimonials.php to read real quotes from published authors.

Submit your manuscript here: https://www.dovepress.com/international-journal-of-general-medicine-journal 